$$
\begin{gathered}
(1-b)^{i}\left[1-(1-b) \frac{n-1-2 i}{2}\right]^{2}>0 \\
(1-b) \frac{n-1}{2}\left[1-(1-b) \frac{n-1-2}{2}-1\right]^{2}>0
\end{gathered}
$$

On aurait pu, de même, constater par substitution des valeurs numériques trouvées pour $\mathbf{b}_{0}$, la légitimité de cette inégalité.

Les tableaux suivants résument les valeurs de $\left(\mathbf{1}-\mathbf{b}_{0}\right)$ et $\left(1-b_{0}\right) \frac{n-1}{2}$.

\begin{tabular}{|c|c|c|c|c|c|}
\hline & $\mathbf{n}=\mathbf{1}$ & $\mathbf{n}=\mathbf{2}$ & $\mathbf{n}=\mathbf{3}$ & $\mathrm{n}=\mathbf{4}$ & $\mathbf{n}=\mathbf{5}$ \\
\hline $\mathrm{a}=0.25$ & 0.750 & 0.875 & 0.905 & 0.923 & 0.940 \\
\hline $\mathrm{n}=0.50$ & 0.500 & 0.708 & 0.793 & 0.835 & 0.860 \\
\hline$a=0.75$ & 0.250 & 0.505 & 0.633 & 0.714 & 0.763 \\
\hline
\end{tabular}

Tableau IV. - Valeurs de $\left(\mathbf{1}-\mathbf{b}_{0}\right)$.

\begin{tabular}{|c|c|c|c|c|}
\hline & $n=2$ & $\mathrm{n}=\mathbf{3}$ & $\mathrm{n}=4$ & $n=5$ \\
\hline$a=0.25$ & 0.935 & 0.905 & 0.886 & 0.883 \\
\hline$a=0.50$ & 0.841 & 0.793 & 0.763 & 0,744 \\
\hline$a=0.75$ & 0.710 & 0.633 & 0.603 & 0.577 \\
\hline
\end{tabular}

Tableau V. - Valeurs de $\left(\mathbf{1}-\mathbf{b}_{0}\right) \frac{\mathfrak{n}-1}{2}$.

En particulier, pour $\mathbf{n}=\mathbf{5}$ et $\mathbf{a}=\mathbf{0 , 7 5}$, nous aurons

$$
\Lambda=\mathfrak{1}_{1} 096
$$

Il en résulte que le rapport $\Lambda=\frac{\mathbf{V}_{\mathrm{e}}}{\mathbf{V}_{\mathrm{B}}}$ deviendrait donc bien, dans ce cas, plus petit que dans celui oú toutes les sections ont une même longueur.

Le tableau suivant résume nos résultats au point de vue de la comparaison des volumes de cuivre nécessaires.

Hypothèse. Cas d'une égale perte de puissance pour les

\begin{tabular}{|c|c|c|}
\hline & Valeurs de $\Lambda$ & $\begin{array}{l}\text { Economie en \% par rap. } \\
\text { port a a la tension e efl } \\
\text { sans survoltage. }\end{array}$ \\
\hline $\begin{array}{c}\text { Sections } \\
\text { de } \\
\text { grandeur égale }\end{array}$ & 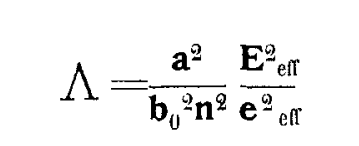 & $\frac{\mathbf{a}^{2}}{\mathbf{b}_{0}{ }^{2} \mathbf{n}^{2}}$ \\
\hline $\begin{array}{l}\text { Sections de } \\
\text { longueurs } \\
\mathrm{L} \alpha_{1}-\mathrm{L} \alpha_{\mathrm{n}}\end{array}$ & 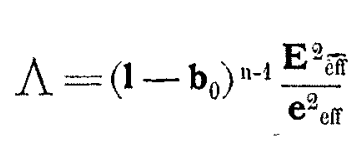 & $\left(s-b_{0}\right)^{n-1}$ \\
\hline $\begin{array}{c}\text { A la tension } \\
\text { eff } \\
\text { sans survoltage }\end{array}$ & $\Lambda=\frac{\mathbf{E}^{2}{ }_{\text {en }}}{\mathbf{e}^{2}{ }_{\text {en }}}$ & 1 \\
\hline
\end{tabular}
tensions $\mathbf{E}_{\text {eff }}$ et $\mathbf{e}_{\text {eff }}$ à la tête de chaque section.

Tableau VI. - Equation définissant $\mathbf{b}_{0} \cdot\left\{\frac{\mathbf{a}}{\mathbf{b} \Sigma}=1\right.$.

(A suivre).

BARBHLLION, Ingénieur-Electricien, Maitre de Conférences à l'institut Electrotechnique de Grenoble.

\section{Le transport de l'énergie par courants alternatifs \\ à intensité constante}

\section{Système usitè aux Etats-Unis pour l'éciairage public}

Dans le premier numéro de La Houille Blanche, M. Rigollot, qui professe avec tant d'autorité le Cours de Physique industrielle à l'Université de Lyon, nous exposait le système de distribution de l'énergie par courants continus à intensité constante et qui a reçu des applications très réussies dans de nombreux transports de force, grâce à l'habileté de mécanicien parfait de $M$. Thury, le sympathique directeur de la Compagnie de l'Industrie Electrique de Genève.

Quoique la mode n'en soit guère maintenant aux transports de force par courants continus, les courants polyphasés offrant des avantages incontestables, il n'en est pas moins vrai que lorsque les frais de premier établissement et les dépenses d'exploitation doivent avant tout être réduits, le srstème continu série, peut lutter avantageusement et même l'emporter sur le terrain économique.

Dans un ordre d'idèes analogues, nous allons parler aujourd'hui, d'un système de distribution de l'énergie par courants alternatifs mono ou polyphasés à intensité constante. Ce système, dont nous ne connaissons pas d'applications en Europe,est par contre généralementusité aux EtatsUnis pour l'éclairage public par lampes à arc en vase clos: nous l'avons vu très souvent fonctionner et, disons le tout de suite, donner d'excellents résultats.

Dans ce mode de distribution à courants alternatifs, il s'agit, comme dans le cas des courants continus, de maintenir constante l'intensité en modifiant le voltage. On y arrive très simplement au moyen d'un transformateur spécial qui maintient constante l'intensité du courant alternatif, dans le circuit sur lequel il est branché, tout en permettant, si on le désire, d'élever ou d'abaisser la tension du courant fourni par le circuit d'alimentation. Cet appareil joue donc un rôle absolument analogue au régulateur de $\mathrm{M}$. Thury agissant sur le rhéostat d'excitation ou sur le calage des balais de la dynamo génératrice; il est d'un usage constant dans les secteurs d'éclairage des villes où l'on emploie des lampes à arc en vase clos branchées en série sur un courant alternatif d'intensité constante.

Le primaire du transformateur est connecté directement sur la ligne monophasée ou polyphasée. Les lampes d'utilisation sont placées sur le secondaire soit toutes en série sur un seul circuit, soit sur deux circuits, ainsi que le montre le schéma ci-joint qui indique en même temps le montage du transformateur.

Le principe de l'appareil lui-même est des plus simples (1). Il se compose d'un circuit magnétique à trois

(I) La plupart des renseignements que nous publions aujourd'hui nous ont été communiqués avec beaucoup d'amabilité par M. C. P. Steinmetz, l'éminent savant américain, au cours de la visite faite en août dernier, aux ateliers de construction de la Général Electric Cóa Schenectady, par la mission française de la Société Internationale des électriciens. 
branches réunies par des culasses; autour du noyau central A sont disposés deux bobines $B^{1}$ et $B^{2}$ comportant chacune le nombre de spires nécessaires. La bobine $B^{1}$ qui est reliée au circuit d'alimentation est fixe; elle est mainteue contre la culasse inférieure du transformateur; la bobine $B^{2}$ est au contraire mobile et peut se déplacer sur le noyau central; à cet effet, elle est reliée par des cables souples aux bornes des circuits d'utilisation. Cette bobine est suspendue à l'extrémité d'un levier $L$ reposant sur des couteaux et portant à son autre extrémité un contre-poids $P$. Ce contre-poids est réglé de telle sorte qu'il équilibre le poids de la bobine $B^{2}$ diminué de la répulsion électro-dynamique qui s'exerce entre $\mathrm{B}^{1}$ et $\mathrm{B}^{2}$ lorsque ces bobines sont parcourues par le courant normal. Quand l'appareil ne fonctionne pas, la bobine $\mathrm{B}^{2}$ se trouve à fond de course près de la bobine fixe; lorsqu'on ferme le circuit secondaire, les forces répulsives agissantes font remonter la bobine mobile et l'équilibre s'établit lorsqu'elle a atteint la position correspondant au

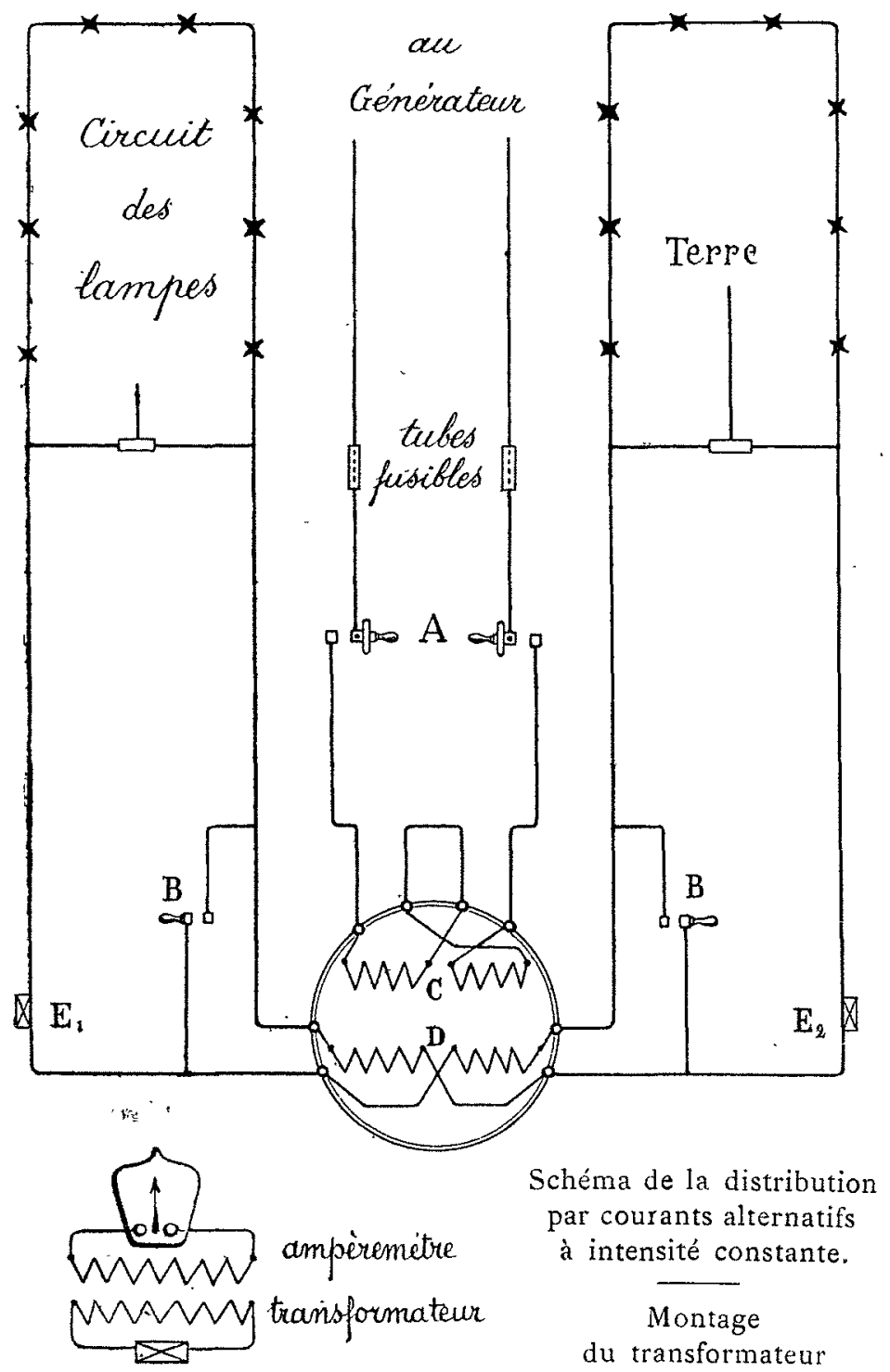

\section{A. Interrupteur primaire. \\ B. Commutateur de court-circuit. \\ $C$. Bobines primaires. - D. Bobines secondaires. $E^{1} E^{2}$. Prises de courant.}

L'ampéremètre transformateur figuré sur le côté sert à mesurer le courant dans les circuits secondaires; il suffit pour cela de connecter le primaire du petit transformateur sur lequel il est monté aux prises de courant $\mathrm{E}^{1}$ ou $\mathrm{E}^{2}$. courant pour lequel le contre-poids a été réglé. Si le courant tend à diminuer dans le circuit d'utilisation, les forces répulsives entre bobines diminuent, $\mathrm{B}^{2}$ se rapproche de $\mathrm{B}^{1}$ et, la force électro-motrice induite dans $B^{2}$ augmentant, le courant reprend sa valeur normale; l'inverse se passe si le courant tend à augmenter.

Ces appareils sont construits par la Général Electric Cu suivant cinq types correspondant à $25,35,50,75$ et 100 lampes. Les petits transformateurs de 25 et 35 lampes sont exactement du modèle décrit ci-dessus; quand aux autres, ils ont la même forme d'armature, mais ont deux séries de bobines, deux primaires et deux secondaires. Les deux bobines primaires sont fixes et situées aux extrémités supé rieure et inféricure de l'armature; les deux secondaires sont libres de se mouvoir verticalement le long de la partie centrale. L'ensemble de l'appareil est complètement plongé dans l'huile contenue dans une cage de fer.

Ces transformateurs règlent le courant, depuis la pleine charge jusqu'à $1 / 3$ de leur capacité. Convenablement réglés, ils maintiennent le courant normal constant à I/ ro d'ampère près en plus ou en moins, depuis la pleine charge jusqu'au tiers de cette charge. La Général Electric Co prétend qu'ils peuvent fonctionner plusieurs heures sur un court circuit sans que l'échauffement devienne dangereux : nous donnons l'affirmation pour ce qu'elle vaut, n'ayant pas eu l'occasion de la vérifier.

Le rendement serait, toujours d'après les essais de la Général Electric $\mathrm{C}^{0}$, de $96 \%$ pour un transformateur de de 100 lampes à arc en vase clos et de 6,6 ampères: il ne serait que de $94,5 \%$ pour un transformateur de 25 foyers. Le facteur de puissance à pleine charge est compris entre 0,76 et 0,78 sur un circuit de lampes de divers modèles.

Les dynamos qui fournissent le courant alternatif d'intensité constante nécessaire à la distribution, sont du système Brush et Thomson. La tension est essentiellement variable et est maximum lorsque toutes les lampes du même circuit sont allumées en même temps.

Voici deux exemples de ces machines:

Station centrale de Washington: 6 dynamos Brush de 9,6 ampères constants et de tension maxima 6,200 volts.

Sous-station de Buffalo, alimentée par l'usine génératrice des chutes du Niagara à 35 kilomètres : i 5 dynamos Brush, commandées chacune directement par un moteur synchrome dont voici les données :

\section{50 kilowatts,}

Moteur synchrone $\left\{\begin{array}{l}6 \text { pôles, } \\ 500 \text { tours par minute, }\end{array}\right.$

352 volts, 25 cycles.

Machine Brush.... $\left\{\begin{array}{l}9,6 \text { ampères constants, } \\ 6.250 \text { volts maximum, } \\ 3 \text { circuits en parallèle sur l'enduit. }\end{array}\right.$

L'éclairage des rues tient une place considérable dans l'exploitation des stations centrales américaines et les dépenses d'entretien des arcs à air libre seraient farticulièrement élevées.

Pendant plus de la moitié de l'année, il faut éclairer de Io à 12 heures par nuit et comme une paire de crayons ne dure pas plus de 6 à 8 heures, leur remplacement s'impose 
en pleine marche pour les arcs simples. L'adoption de l'arc en vase clos, dont la durée des crayons est environ 15 fois plus grande, représente donc une véritable économie; aussi leur emploi est-il aujourd'hui général. Le tableau suivant dû à M. Alton Adams montre comment l'arc en vase clos a remplacé l'arc simple et l'arc double; il se rapporte à l'ensemble des stations centrales de l'Etat de Massachusetts.

\begin{tabular}{|c|c|c|c|}
\hline EXERCICES & ARCS SIMPLES & ARGS DOUBLES & $\begin{array}{c}\text { ARCS EN VASE } \\
\text { CLOS }\end{array}$ \\
\hline $1887-1888$ & 4.392 & 2.187 & $n$ \\
\hline $1890-1891$ & 5.753 & 6.115 & $n$ \\
\hline $1893-1894$ & 7.286 & 9.316 & $"$ \\
\hline $1896-1897$ & 7.385 & 12.144 & 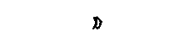 \\
\hline $1897-1898$ & 7.485 & 12.362 & $"$ \\
\hline $1898-1899$ & 5.096 & 11.857 & 4.300 \\
\hline $1899-1900$ & 2.915 & 8.452 & 11.988 \\
\hline
\end{tabular}

Quelles sont donc les raisons qui expliquent la préférence des américains pour le système de distribution par courants alternatifs d'intensité constante et pour les lampes à arc en vase clos?

Les raisons ne sont pas d'ordre technique, mais d'ordre économique et commercial, comme il arrive souvent en Amérique.

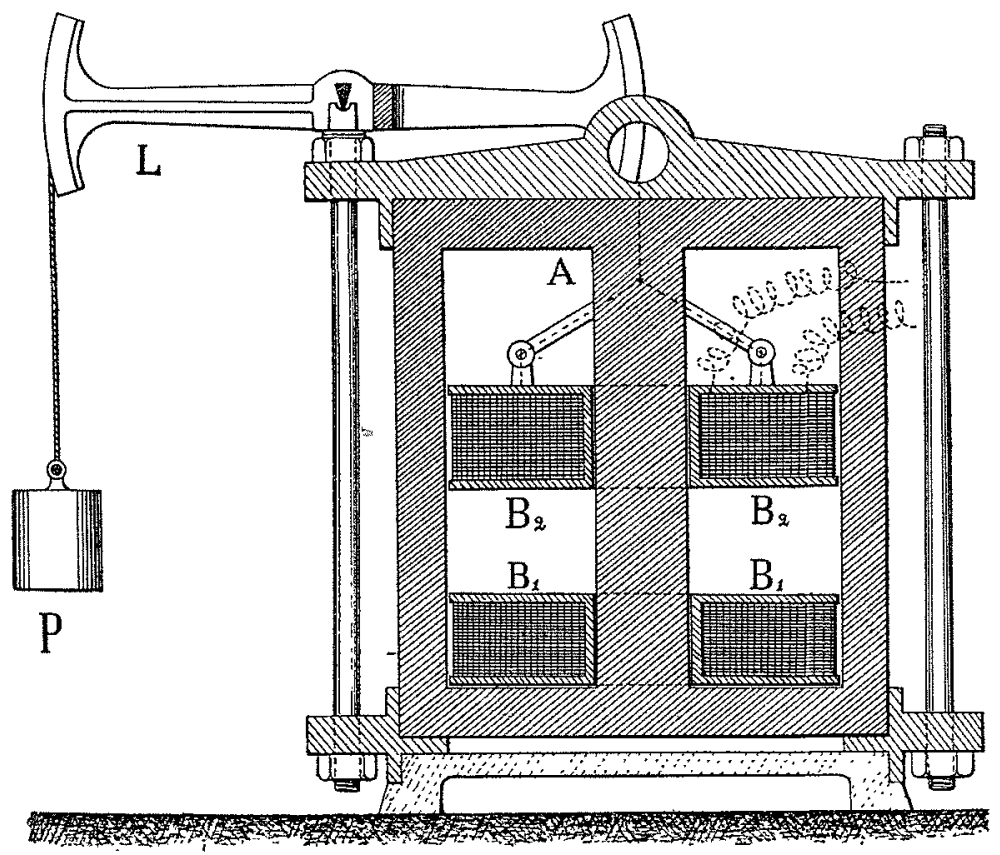

Transformateur pour courants a!ternatifs à intensité constante.

Le fonctionnement de ces transformateurs régulateurs est, il est vrai, excellent, mais le rendement des dynamos génératrices du systeme Brush est faible; les collecteursbagues s'usent rapidement par suite d'étincelles inévitables, Le rendement lumineux des lampes en vase clos est mauvais. surtout lorsque les chàrbons ne sont pas d'une qualité irréprochable, car alors le verre se recouvre de petites paillettes noires qui finissent par former un écran opaque. A ces inconvénients, il faut opposer les avantages économiques suivants :
Nombre de stations centrales américaines doivent compter l'éclairage public des rues comme une fraction importante de leur clientèle et ont dû disposer des groupes électrogènes destinés uniquement à alimenter ce service. Le nombre de kilomètres de rues à éclairer est toujours considérable; il faut donc, si on veut diminuer les frais d'entretien et de personnel, avoir de gros foyers lumineux aussi éloignés que possible, et pouvant fonctionner toute la nuit sans aucune surveillance et c'est ce qui se trouve, en partie tout au moins, réalisé par les lampes en vase clos. Quant à la question du rendement de la distribution, elle est secondaire pour les américains qui peuvent avoir à Buffalo, par exemple, le gaz naturel à o fr. 0484 le mètre cube, ce gaz venant de Bradfort (Pensylvanie), à une distance de r 90 kilomètres; le charbon lui-même est bon marché puisqu'il revient à la station centrale de Philadelphie à q fr. $7^{5}$ la tonne, se décomposant ainsi :

$9 \mathrm{fr}$. la tonne prise à la mine.

o fr. 6o pour le transport par eau qui est de 150 kilomètres.

o fr. 15 pour le déchargement à l'usine au 'moyen d'une noria.

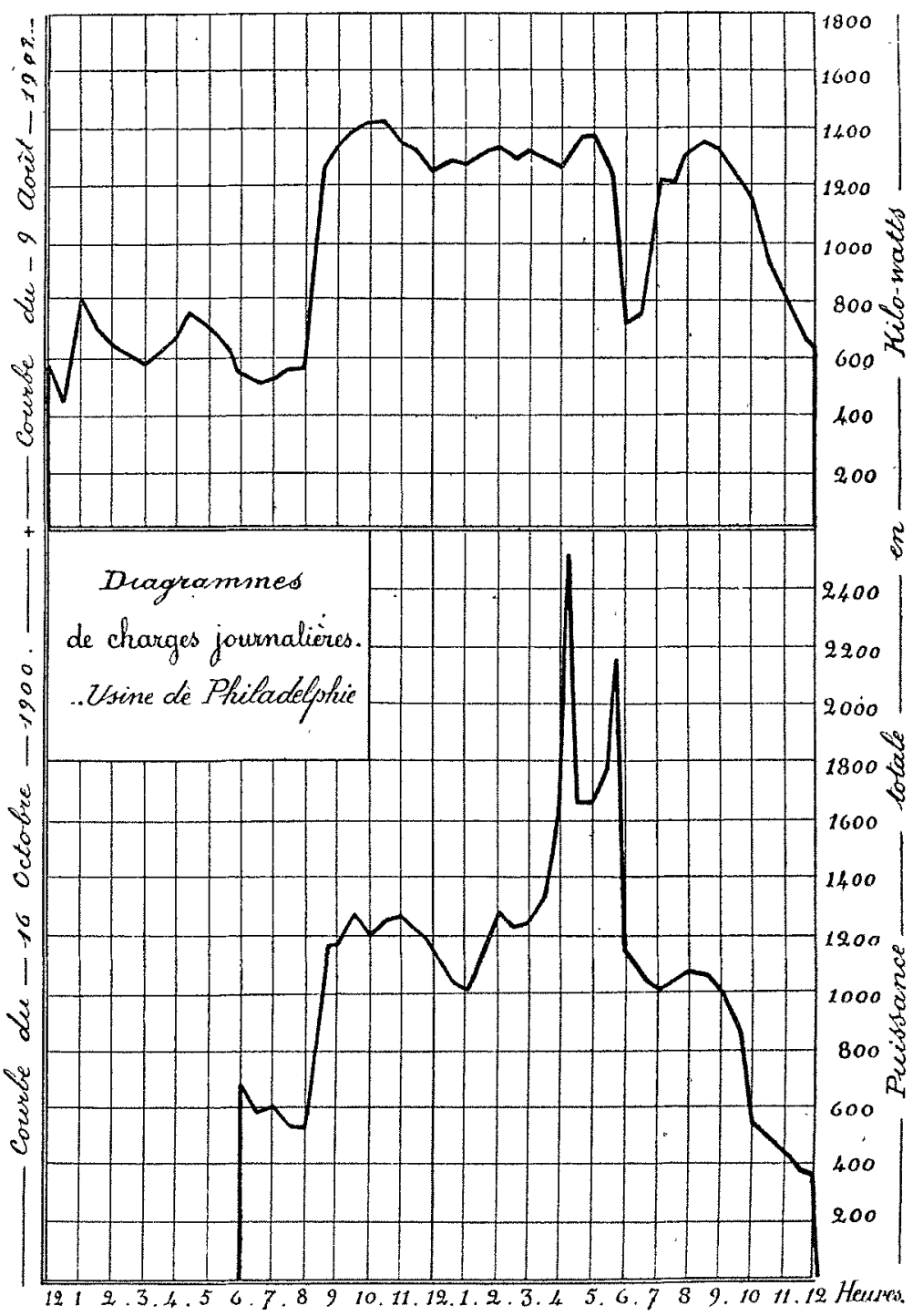

Nous terminons en donnant deux courbes de charge journalière prises à des époques assez différentes, 9 août igo I, I 6 octobre Igoo, et se rapportant à l'usine génératrice de Philadelphie. Dans la seconde, la pointe très haute qui se produit vers 4 heures, indique le moment où les bureaux 
de commerce, banques, administrations, etc., allument leurs lampes. Vers 5 heures, les bureaux sont fermés et c'est le tour des restaurants, hôtels, etc., qui produisent la seconde pointe vers 5 heures trois quarts.

La première courbe se rapporte à la saison d'été; les deux pointes n'existent pas et l'on voit une allure plus régulière.

En de prochains articles, nous décrivons l'utilisation électrique des chutes du Niagara.

\section{J . Courbier,}

Ancien élève de l'Ecole Polytechnique et de l'Ecole Supérieure d'Electricité de Paris Licencie en Droit.

\section{Réflexions sur les études préliminaires à l'aménagement des chutes d'eau}

\section{Essai de classification des chutes}

10 Que faut-il entendre par haute, moyenne et basse clute? Y a-t-il intérêt à conserver ces expressions?

A prendre les choses du point de vue de la mécanique rationnelle, ces expressions imprécises sont sans utilité, l'indication de la mesure métrique du débit et de la hauteur suffisant à différencier les chutes entre elles. Aussi les traités didactiques, tout au moins ceux qui sont à la disposition du commun des mortels, traitent-ils cette question par prétérition.

Cependant les auteurs qui ont dû exposer la question au public ont du lui parler sa langue.M. Aristide Bergès (opuscule La Houille Blanche, page 7 , ligne i 3 ) emploie l'expression haute chute ce qui implique l'usage de toute la série. Les auteurs de publications industrielles récentes, les constructeurs de machines se servent, comme le grand public, de ces expressions et on doit les adopter quand on veut se faire entendre.

Il convient done d'en préciser le sens.

Tout d'abord la valeur de ces dénominations ressort-elle nettement de l'usage qui en est actuellement fait ?

Voici, par exemple, le savant directeur d'une très importante maison de constructions, qui a un renom universel, que lisons-nous dans la notice qu'il a rédigée pour diriger ses clients, dans le choix d'une turbine ou d'une roue?

"Nous divisons les chutes en quatre classes:

I $^{\circ}$ Les basses chutes, dont la hauteur est comprise entre o m. 5 o et $3 \mathrm{~m}$.

$2^{\circ}$ Les morennes chutes, entre $3 \mathrm{~m}$. et $8 \mathrm{~m}$.

30 Les hautes chutes, entre $8 \mathrm{~m}$. et $12 \mathrm{~m}$.

$4^{0}$ Les très haules chutes, depuis I $2 \mathrm{~m}$. et au-delà. "

Cette classification semble calquée sur l'ancicnne répartition des machines à vapeur entre les types de machines à basse, moyenne et haute pression, laquelle prêtait fortement à l'arbitraire, comme chacun sait, et variait selon les auteurs et les constructeurs.

A côté de cette première classification nous devons citér l'opinion d'une maison de constructions étrangère, et non des moindres. Pour elle les hautes chutes ne commencent qu'au delà de $100 \mathrm{~m}$.

M. Rateau, ingénieur des Mines, au Congrès Interna. tional de Mécanique de 1900 , dit qu'on est "assez généralement d'accord pour qualifier de petites chutes, celles qui sont inférieures à $6 \mathrm{~m}$. et de grandes celles qui dépassent $40 \mathrm{~m} . "$

Enfin, M. de la Brosse, ingénieur en chef des Ponts et Chaussées, dans une étude (I) récente qualifie de chutes faibles (quelques mètres) des chutes ayant jusqu'à $12 \mathrm{~m}$. de haut et de chute d'importance morenne des chutes de $60 \mathrm{~m}$. à 7 o $\mathrm{m}$.

Il ne qualifie pas les chutes de $500 \mathrm{~m}$. à $600 \mathrm{~m}$. et il n'indique pas non plus, encore qu'on s'en doute, dans quelle catégorie il range celles de $20 \mathrm{~m}$. à $40 \mathrm{~m}$.

Sans qu'il soit nécessaire de prolonger ces citations, on avouera qu'il est embarrassant de choisir et qu'il est bien désirable de trouver un moyen de sortir de l'indécision, ne serait-ce que pour s'entendre.

Nous croyons avoir trouvé un pareil moyen, mais avant de le proposer il convient d'élucider un point spécial, qui servira à préciser nos conventions.

\section{$2^{\circ}$ Quelle limite extrême de hauteur peut-on assigner actuellement aux plus hantes chutes?}

Théoriquement, il n'y a pas de limite supérieure à fixer aux hauteurs de chute; mais, en pratique, il semble qu'on puisse en fixer une en s'aidant de considérations rationnelles.

Pour capter les chutes on se sert de tuyaux et, afin d'allier la solidité à la légèreté, on les fait souvent en métal. Or, la résistance spécifique des métaux aux efforts d'extension auxquels on peut les soumettre n'est pas indéfinie. C'est cette résistance que nous utiliserons en premier lieu, pour assigner une limite pratique à la hauteur des colonnes d'eau à mettre en cuvre.

Actuellement la métallurgie fournit au commerce des fontes capables de résister couramment à des efforts de traction de I 55 par millimètre carré de section (2) du métal. Or, on sait d'après les théorèmes de Lamé, que si $t$ représente la tension moyenne par unité de surface de la section transversale de la matière du tube et $p$ la pression qui s'exerce sur l'unité de la surface cylindrique intérieure au même endroit, on a entre ces deux forces l'équation:

$$
\mathrm{t}-\mathrm{p}=\mathrm{c}^{\mathrm{tc}}=\mathrm{a}
$$

$a$ est la tension initiale du métal quand la pression $p$ est nulle $\left(a=t-o=t_{0}\right)$. Dans un tuyau simple, plein d'air, la tension

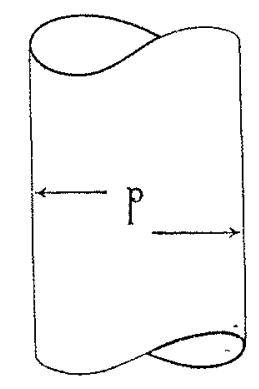
initiale $t_{0}$ est nulle, c'est la valeur de la constante dans ce cas particulier, et nous écrivons :

$$
\mathrm{t}-\mathrm{p}=\mathrm{o}
$$

(1) Les installations hydro-électriques dans la région des Alpes, par R. de la Brosse - Grenoble, Gratier.

(2) Soit $1 / 10$ de l'effort produisant la rupture estimé à $15 \mathrm{~kg}$ par millimètre carré de section pour les fontes ordinaires. Une fabri. cation soignée obtient beaucoup plus. 